Article

\title{
An Intranuclear Sodalis-Like Symbiont and Spiroplasma Coinfect the Carrot Psyllid, Bactericera trigonica (Hemiptera, Psylloidea)
}

\author{
Saptarshi Ghosh ${ }^{1} \oplus$, Noa Sela ${ }^{2} \mathbb{D}$, Svetlana Kontsedalov ${ }^{1}$, Galina Lebedev ${ }^{1}$, Lee R. Haines ${ }^{3} \mathbb{C}$ \\ and Murad Ghanim 1,*(D) \\ 1 Department of Entomology, Institute of Plant Protection, Volcani Center, ARO, HaMaccabim Road 68, \\ P.O. Box 15159, Rishon LeZion 7528809, Israel; sunnysaptarshi@gmail.com (S.G.); \\ nasvetla@yahoo.com (S.K.); galinal@volcani.agri.gov.il (G.L.) \\ 2 Department of Plant Pathology and Weed Research, Institute of Plant Protection, Volcani Center, ARO, \\ HaMaccabim Road 68, P.O. Box 15159, Rishon LeZion 7528809, Israel; noa@volcani.agri.gov.il \\ 3 Department of Vector Biology, Liverpool School of Tropical Medicine, Pembroke Place, Liverpool L3 5QA, \\ UK; Lee.Haines@lstmed.ac.uk \\ * Correspondence: ghanim@agri.gov.il
}

Received: 4 April 2020; Accepted: 4 May 2020; Published: 8 May 2020

\begin{abstract}
Endosymbionts harbored inside insects play critical roles in the biology of their insect host and can influence the transmission of pathogens by insect vectors. Bactericera trigonica infests umbelliferous plants and transmits the bacterial plant pathogen Candidatus Liberibacter solanacearum (CLso), causing carrot yellows disease. To characterize the bacterial diversity of B. trigonica, as a first step, we used PCR-restriction fragment length polymorphism (PCR-RFLP) and denaturing gradient gel electrophoresis (DGGE) analyses of $16 \mathrm{~S}$ rDNA to identify Sodalis and Spiroplasma endosymbionts. The prevalence of both symbionts in field-collected psyllid populations was determined: Sodalis was detected in $100 \%$ of field populations, while Spiroplasma was present in $82.5 \%$ of individuals. Phylogenetic analysis using $16 \mathrm{~S}$ rDNA revealed that Sodalis infecting B. trigonica was more closely related to symbionts infecting weevils, stink bugs and tsetse flies than to those from psyllid species. Using fluorescent in situ hybridization and immunostaining, Sodalis was found to be localized inside the nuclei of the midgut cells and bacteriocytes. Spiroplasma was restricted to the cytoplasm of the midgut cells. We further show that a recently reported Bactericera trigonica densovirus (BtDNV), a densovirus infecting B. trigonica was detected in $100 \%$ of psyllids and has reduced titers inside CLso-infected psyllids by more than two-fold compared to CLso uninfected psyllids. The findings of this study will help to increase our understanding of psyllid-endosymbiont interactions.
\end{abstract}

Keywords: psyllid; Bactericera trigonica; Candidatus Liberibacter solanacearum; secondary endosymbiont; Sodalis; Spiroplasma; intranuclear bacteria

\section{Introduction}

Symbiotic bacterial associations with insect hosts can be either obligate or facultative in nature. Obligate bacterial symbionts are always vertically transmitted, are mostly located in a specialized structure called the bacteriome and typically co-speciate with their insect hosts [1,2]. Obligate symbionts share metabolic pathways with their hosts and synthesize essential amino acids and vitamins [3]. Facultative bacterial symbionts have been more recently acquired by their insect hosts, colonize multiple insect tissues and organelles and can be transmitted either vertically or horizontally $[2,4]$. Facultative symbionts provide conditional benefits to their hosts such as heat tolerance [5,6], defense against pathogens [7,8] and parasites [9], resistance to pesticides [10], and reproductive advantages 
such as increased fecundity and adult emergence [11]. Facultative symbionts can also influence the insect's ability to transmit insect-borne pathogens $[12,13]$.

Psyllids (Hemiptera, Psylloidea), also known as plant lice, are tiny, phloem-sucking, plant-specific insects of enormous agricultural importance as they transmit devastating diseases to food crops. A gamma-proteobacterial symbiont, Candidatus Carsonella ruddii, is harbored inside the bacteriocytes of all psyllid species [14,15]. Alongside Candidatus Carsonella, a diverse group of facultative symbionts such as Arsenophonus and Sodalis (gamma-proteobacteria), as well as Rickettsia and Wolbachia (alpha-proteobacteria) contribute to the psyllid endosymbiotic microflora [14,16-18]. Bactericera trigonica (B. trigonica) is a psyllid species infesting umbelliferous crops in the Mediterranean and Middle East. B. trigonica transmits the emerging, phloem-restricted plant pathogen Candidatus Liberibacter solanacearum (CLso) (haplotype D), which causes significant economic losses to crops such as carrots, celery and parsley [19-22]. Carrots are a major vegetable export from Israel to European markets; however, since 2009, an epidemic of CLso transmitted by B. trigonica has caused significant financial shortfalls for those in the carrot industry in Israel and Europe. Over $80 \%$ of the carrot plants with yellow symptoms and 10\% of asymptomatic ones tested positive for CLso in Israel in 2015 [23].

CLso is transmitted by B. trigonica in a circulative, persistent manner; ingested bacteria migrate across insect midgut barriers to reach the hemolymph and then penetrate the salivary glands before the insect can inoculate the plant phloem upon feeding [21,22]. Several insect-borne plant pathogens with tissue-specific lifecycles can interact with endosymbionts to influence transmission efficiency. For example, endosymbionts of the whitefly Bemisia tabaci and of several aphid species enhance the transmission of pathogenic, insect-borne plant viruses by protecting the viruses from the hostile environment (up-regulated immune factors) in the host hemolymph [13,24]. In contrast, the presence of a symbiont, Wolbachia spp., reduces the capacity of mosquitoes to transmit Dengue virus, which clearly demonstrates how these "defensive" endosymbionts can be exploited to control virus transmission $[25,26]$.

The characterization of the endosymbiotic diversity and their niche inside $B$. trigonica is a first step to understanding their direct or indirect interactions with CLso. In this study, we investigated the bacterial endosymbionts inside B. trigonica and identified two facultative symbionts, Spiroplasma $s p$. and Sodalis-like bacteria that colonize B. trigonica alongside Carsonella sp., the primary symbiont. Additionally, we also describe the prevalence and dynamics of the recently reported Bactericera trigonica densovirus (BtDNV) [27] inside the psyllids with and without CLso. We also localize the symbionts within insect tissues to understand their spatial distribution and possible influence on host physiology. Moreover, we show that psyllids infected with CLso have reduced titers of BtDNV compared to psyllids free of CLso.

\section{Materials and Methods}

\subsection{Insect Collection and Culture}

B. trigonica-CLso-infected and uninfected populations-were collected from carrot fields in Southern Israel during the summer of 2015. The CLso infection status (uninfected/infected) of collected psyllid populations was determined by the qPCR (Table 1) of celery plants used to rear the psyllids after 2 months from the release of insects. Laboratory CLso (haplotype D)-infected (100\% infection, reared on CLso infected plants) and uninfected populations (reared on uninfected plants) were maintained in separate cages on organically raised celery (Apium graveolens, variety Spartacus, which is a suitable host for both CLso and B. trigonica) under controlled environmental conditions $\left(25^{\circ} \mathrm{C}, 60 \%\right.$ R.H, $14 \mathrm{hL}$ : $10 \mathrm{hD}$ ). The presence and absence of CLso from psyllid populations were routinely confirmed every 3 months by performing qPCR on insects and the celery plants used for rearing. 


\subsection{PCR-RFLP and Denaturing Gradient Gel Electrophoresis (DGGE) of $16 S$ rDNA}

Total insect DNA was extracted from surface-sterilized (immersion in 70\% ethanol for $5 \mathrm{~min}$ ) individual CLso-infected and uninfected insects by the CTAB extraction method [28]. Briefly, each insect was homogenized in $500 \mu \mathrm{L}$ of CTAB buffer containing $1 \mu \mathrm{L}$ of $\beta$-mercaptoethanol and then incubated at $37^{\circ} \mathrm{C}$ for $45 \mathrm{~min}$. Equal volumes of phenol:chloroform:isoamyl alcohol (25:24:1) were added, and then samples were centrifuged at $1300 \mathrm{rpm}$ for $10 \mathrm{~min}$. To the supernatant, equal volumes of isopropanol were added, and the DNA was allowed to precipitate at $-20^{\circ} \mathrm{C}$ for $1 \mathrm{~h}$. DNA pellets were obtained by centrifugation at $1300 \mathrm{rpm}$ for $10 \mathrm{~min}$ followed by washing with $70 \%$ ethanol. The pellets were air-dried and dissolved in $50 \mu \mathrm{L}$ DNase free water.

A $1.5 \mathrm{~kb}$ fragment of the eubacterial $16 \mathrm{~S} \mathrm{rDNA}$ was amplified by using the universal eubacterial primers fD1 (5' AGAGTTTGATCCTGGCTCAG 3') and rP1 (5' GGTTACCTTGTTACGACTT 3') [29] with thermal cycling conditions of $95^{\circ} \mathrm{C}$ for $3 \mathrm{~min}$, followed by 35 cycles of $95{ }^{\circ} \mathrm{C}$ for $20 \mathrm{~s}, 52{ }^{\circ} \mathrm{C}$ for $30 \mathrm{~s}$ and $72{ }^{\circ} \mathrm{C}$ for $2 \mathrm{~min}$. The PCR products were gel-purified and cloned into pGEM-T easy vector (Promega, U.S.A). Clones containing the insert were confirmed by colony PCR with T7F and SP6 promoter primers. Colony PCR products (300 colonies) were digested with Taq I (ThermoFisher Scientific) and visualized on $2 \%$ agarose gels. Five clones each for unique restriction fragment length polymorphism (RFLP) profile patterns were sequenced (HyLabs, Rehovot, Israel) for both directions.

Alternatively, a $500 \mathrm{bp}$ fragment of the eubacterial 16S rDNA was PCR amplified using primer set 341F with GC clamp (5'CGCCCGCCGCGCCCCGCGCCCGTCCCGCCGCCCCC GCCCGCCTACGGGAGGCAGCAG 3') and 907R (5' CCGTCAATTCMTTTGAGTTT 3') [30] followed by denaturing gradient gel electrophoresis (DGGE) on a vertical gel containing $8 \%$ polyacrylamide with a linear gradient of denaturants increasing from $0 \%$ to $60 \%$. Electrophoresis was performed in $1 \times$ TAE at $200 \mathrm{~V}$ for $4 \mathrm{~h}$ at $60^{\circ} \mathrm{C}$. Gels were stained with ethidium bromide and photographed on a UV trans-illuminator. The DGGE bands were excised, purified and sequenced (HyLabs, Rehovot, Israel).

Table 1. Primer and probe sequences used in this study. CLso: Candidatus Liberibacter solanacearum.

\begin{tabular}{|c|c|c|c|}
\hline Primer & Target & Sequence $\left(5^{\prime}-3^{\prime}\right)$ & Product Size \\
\hline Actin-F & \multirow{2}{*}{ B. trigonica actin gene } & AGATGACCCAGATCATGTTTGA & \multirow{2}{*}{$160 \mathrm{bp}$} \\
\hline Actin-R & & AGGGCGTAACCTTCATAGATG & \\
\hline Lso-F & \multirow{2}{*}{ CLso omp A gene } & ССАТАТССАААТTTCAAAGAACC & \multirow{2}{*}{$152 \mathrm{bp}$} \\
\hline Lso-R & & ATGCCACGTGAAGGTTTGAT & \\
\hline Sod-F1 & \multirow{2}{*}{ Sodalis GroEL gene } & CCAAAGACGGCGTATCAGTT & \multirow{2}{*}{$160 \mathrm{bp}$} \\
\hline Sod-qR2 & & GCCTTCGTTGACGATAGACT & \\
\hline Spir-F & \multirow{2}{*}{ Spiroplasma 16S rDNA } & CTGCCTCATGGCAACACTTA & \multirow{2}{*}{$170 \mathrm{bp}$} \\
\hline Spir-R & & TTTCATGTGTAGCGGTGGAA & \\
\hline Denso-qF & \multirow{2}{*}{ BtDNV VP4 gene } & CACCGAGAACACGCACTTTG & \multirow{2}{*}{$149 \mathrm{bp}$} \\
\hline Denso-qR & & GACCAAAACTCTGGAGGGCA & \\
\hline Probe & Target & Sequence $\left(5^{\prime}-3^{\prime}\right)$ & Reference \\
\hline Carsonella & $16 \mathrm{~S}$ rDNA & Сy3-CGCGACATAGCTGGATCAAG & [31] (pB-1664) \\
\hline CLso & $16 \mathrm{~S}$ rDNA & Су3-GCCTCGCGACTTCGCAACCAAT & This study \\
\hline Sodalis & $16 \mathrm{~S}$ rDNA & Су3/Су5-GTTAССCGCAGAAGAAGCAC & This study \\
\hline Spiroplasma & 16S rDNA & Сy5-TTTCATGTGTAGGGGTGGAA & This study \\
\hline
\end{tabular}

\subsection{Prevalence of Symbionts in Field Collected B. trigonica}

Forty psyllid samples (20 males and 20 females) collected from different carrot fields in the south/north of Israel (Saad, Khavat Eden) were tested individually for the presence of secondary symbionts and a densovirus infecting B. trigonica (BtDNV) [27] using specific primers (Table 1). A primer amplifying the actin gene of B. trigonica (designed using actin sequences in Genbank, KT185024.1 and XM_008470468.2) was used as an internal control (Table 1). 


\subsection{Relative Quantification of Symbionts in CLso-Infected/Uninfected Psyllids}

Relative amounts of Sodalis, Spiroplasma and BtDNV quantified by qPCR was compared between adult psyllids reared on CLso-infected and uninfected celery plants. Sodalis, Spiroplasma and BtDNV infected $100 \%$ of the laboratory psyllid population used in this study. Total DNA was extracted from a pool of five insects (three female and two male, 5-10 days old) by the CTAB extraction method as described previously. Primer sets were designed to amplify specific targets of Sodalis, Spiroplasma and BtDNV (Table 1). Nine replicates of CLso-infected and uninfected samples each were used for the relative quantification of Sodalis and Spiroplasma, while 14 replicates each were used for BtDNV. A primer amplifying the actin gene of $B$. trigonica was used as an internal reference to normalize the symbiont titres. The efficiency of all primers used ranged between $95 \%-103 \%$. The PCR reactions were run in $15 \mu \mathrm{L}$ reactions with $7.5 \mu \mathrm{L}$ of Thermo Scientific Absolute Blue qPCR SYBR Green ROX Mix (Thermo Scientific) and $300 \mathrm{nM}$ of forward and reverse primer each. The cycling conditions were as follows: $95^{\circ} \mathrm{C}$ for $10 \mathrm{~min} ; 40$ cycles of $95^{\circ} \mathrm{C}$ for $10 \mathrm{~s}, 58^{\circ} \mathrm{C}$ for $20 \mathrm{~s}$ and $72{ }^{\circ} \mathrm{C}$ for $30 \mathrm{~s}$; and a melt curve of $60-90{ }^{\circ} \mathrm{C}$ with a holding step for one second for every $0.5{ }^{\circ} \mathrm{C}$ rise. The relative bacteria quantities relative to the actin gene of $B$. trigonica were calculated using the Delta $\mathrm{Ct}$ method. Mean quantities of the symbionts were analyzed using a simple linear model with $\log _{\mathrm{e}}$-transformed data, and statistical inference was based on the results of a one-way analysis of variance (ANOVA).

\subsection{Fluorescent in Situ Hybridization (FISH) and Immunostaining for Bacteria Localization}

Insect internal organs (guts, ovary, fat bodies, bacteriome, testes and salivary glands) from CLso-infected and CLso-free B. trigonica were dissected into $1 \times$ phosphate saline buffer ( $\mathrm{pH}$ 7.2) under a stereomicroscope. Bacteria (CLso, Sodalis and Spiroplasma) were localized in the dissected organs by fluorescent in situ hybridization (FISH) as described in Ghanim et al. (2016) [32]. Briefly, the dissected organs were fixed in Carnoy's fixative (chloroform: ethyl alcohol: acetic acid, 6:3:1, v/v) followed by overnight hybridization with the probes at room temperature.

\subsection{Immunolocalization of Sodalis and CLso}

Sodalis was immunolocalized in B. trigonica using the mouse anti-Sodalis glossinidius GroEL monoclonal antibody [33] (a gift from Prof. Terry Pearson, University of Victoria, Victoria, BC, Canada). Midguts from adult psyllids were dissected in $1 \times$ PBS buffer followed by the immunolocalization of Sodalis using anti-Sodalis GroEL primary monoclonal antibody (1:100) as described in Ghanim et al. (2017) [21].

\subsection{Sequencing and Genome Assembly of the Sodalis-Like Symbiont}

Total nucleic acids were extracted from 20 surface-sterilized CLso-infected adult psyllids by the CTAB method, as described previously. Genomic DNA was sequenced using Illumina HiSeq 2000 system and paired-end libraries, with an average insert size of $300 \mathrm{bp}$, were constructed (BGI, Hong Kong). A total of $61,673,620$ reads were obtained from the pair-ended libraries with a final yield of 61,658,314 million (99.98\%) clean reads after the trimming of adaptor and low-quality reads using the trimmomatic software [34]. Pair-end library reads were assembled using the pipeline a5 assembly [35] and 625,635 contigs were generated with an N50 of $1198 \mathrm{bp}$. The assembly was then integrated into the Contig Assembly of Prokaryotic Draft Genomes Using Rearrangements (Available online: http://genome.cs.nthu.edu.tw/CAR/) pipeline [36] with the Sodalis glossinidius genome (GCF_000010085.1) as a reference.

The Sodalis-like bacterial genome associated with B. trigonica contains 753 contigs. This genome was uploaded to the RAST server for annotation [37] and compared with other Sodalis genomes available in public repositories. Phylogenetic analysis was performed by concatenating all homologous proteins with the aid of the software OrthoFinder version 2.1.2 [38]. The Multiple Sequence Alignment (msa) algorithm from Orthfinder was used to create the phylogenetic trees with 100 bootstraps in phyml 
software version 3.0 (Available online: http://www.atgc-montpellier.fr/phyml/) [39]. The genome of the Sodalis endosymbiont of B. trigonica was uploaded to the NCBI database under the accession GCA_003668825.1.

\section{Results}

\subsection{Identification and Prevalence of Symbionts Associated with B. Trigonica}

The Sanger sequencing of the $16 \mathrm{~S}$ rDNA clones with different RFLP profiles and the bands obtained by DGGE analyses identified Sodalis-like sp., Spiroplasma sp. and CLso by comparing the sequences with other bacterial sequences using the NCBI BLAST algorithm. Sodalis-like bacteria have been previously identified from other psyllids: the eucalyptus psyllid (Blastopsylla occidentalis), potato psyllid (Bactericera cockerelli) and alder psyllid (Psylla floccosa) (Figure 1).

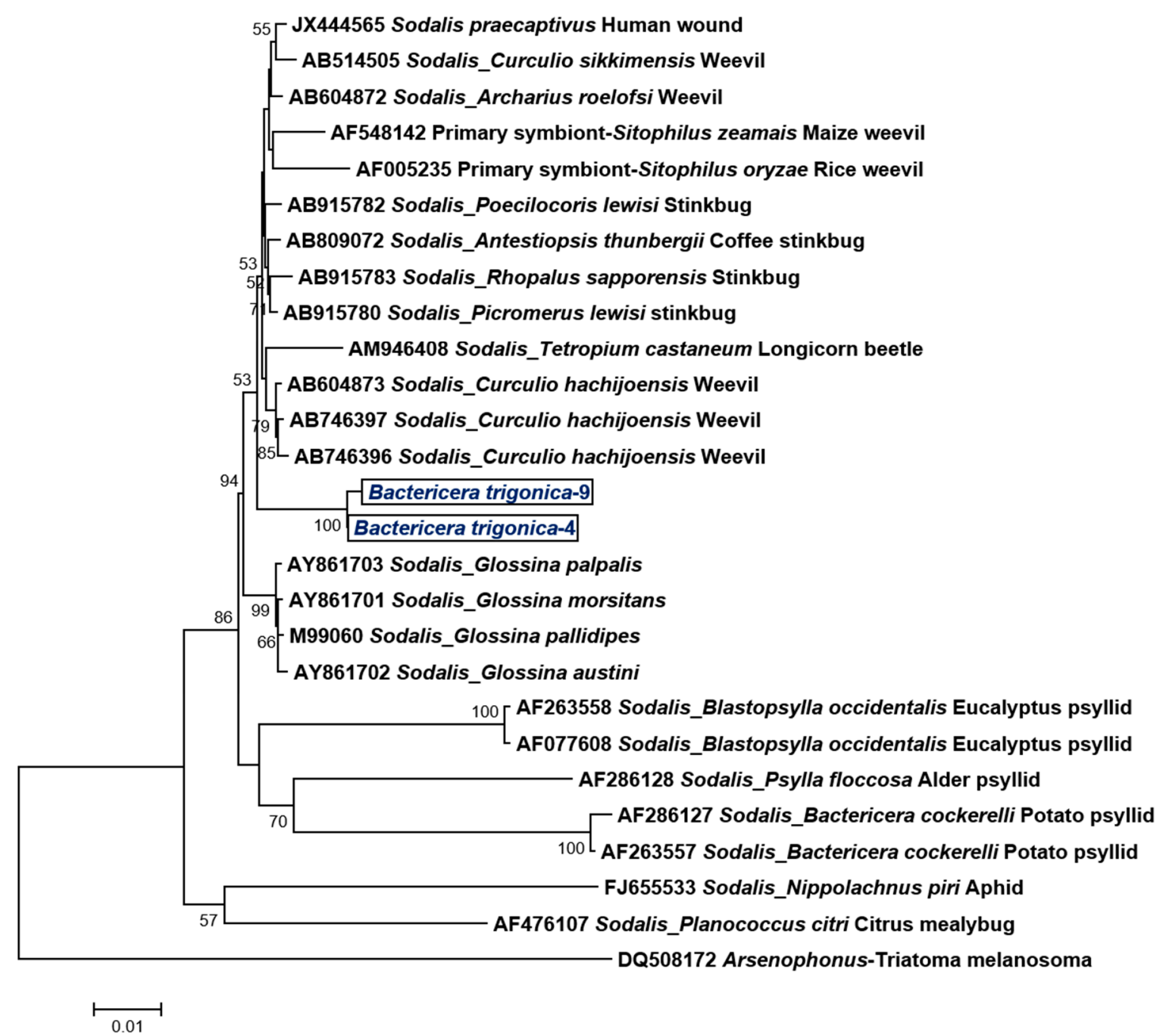

Figure 1. Maximum likelihood phylogenetic tree of nucleotide sequences of Sodalis-like 16S rDNA (1440 bp) from diverse insects by HKY + G + I nucleotide substitution model using MEGA 6. The Sodalis members identified inside $B$. trigonica are indicated inside a text box.

The phylogenetic analysis of the 16S rDNA nucleotide sequences clustered Sodalis infecting B. trigonica with Sodalis species found in weevils, stinkbugs, tsetse flies and a human wound (with $>98 \%$ similarity), but not with the Sodalis spp., which was previously identified from other psyllids or other members of the Sternorrhyncha (aphids, mealybugs) (Figure 1). The phylogenetic 
analysis of the $16 \mathrm{~S}$ rDNA fragment grouped the Spiroplasma infecting B. trigonica closely with Spiroplasma ixodetis Y32 strain (99.3\% nucleotide identity)—a symbiont prevalent in ticks, mosquitoes, pea aphids and ladybird beetles (Figure 2).

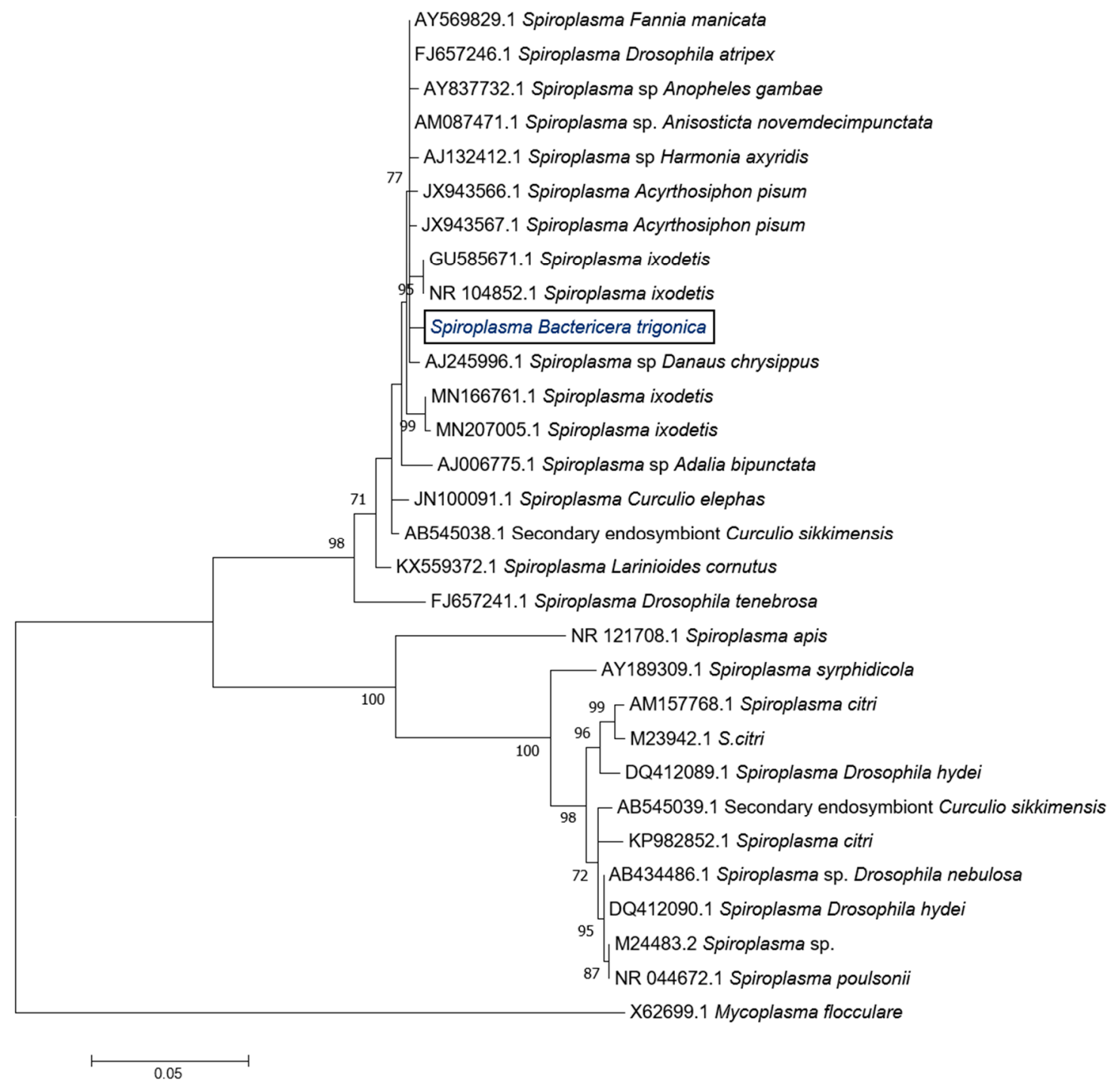

Figure 2. Maximum likelihood phylogenetic tree of $16 \mathrm{~S}$ rDNA nucleotide sequences of Spiroplasma (1193 bp) by T93 + G nucleotide substitution model using MEGA 6 .

The prevalence of the identified symbionts in the B. trigonica were analyzed by PCR. Sodalis was prevalent in $100 \%$ of the field B. trigonica samples tested (40/40), while Spiroplasma was detected in $82.5 \%(33 / 40)$ of the insects tested. We also tested the prevalence of a previously reported densovirus (BtDNV) found to infect B. trigonica in this study. Densovirus (BtDNV) was also detected in $100 \%$ of the B. trigonica samples tested (40/40).

\subsection{Relative Quantification of Symbionts in CLso-Infected/Uninfected Psyllids}

To test whether CLso, the bacterial plant pathogen transmitted by B. trigonica, directly interacts with the identified endosymbionts, we quantified the relative amounts of Sodalis, Spiroplasma and BtDNV in psyllids infected and uninfected with CLso. Relative amounts of the bacterial symbiont, Sodalis (Figure 3A) and Spiroplasma (Figure 3B) did not differ significantly between psyllids infected 
with or free of CLso. However, psyllids infected with CLso had significantly reduced quantities of BtDNV by greater than two-fold $(\mathrm{F}=26.84, p<0.001)$ compared to psyllids free of CLso (Figure 3C).
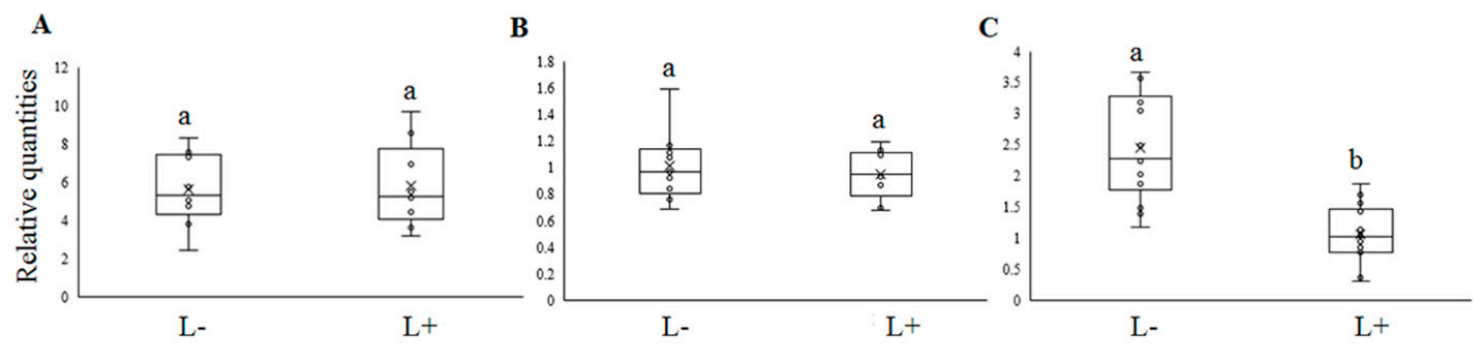

Figure 3. Relative quantities of Sodalis (A), Spiroplasma (B) and BtDNV (C) inside CLso-infected (L+) and uninfected (L-) psyllids. Different lowercase letters indicate significant differences of means $(p<0.001)$.

\subsection{Tissue Localization of Sodalis, Spiroplasma and CLso within B. trigonica}

Using FISH, Sodalis DNA was localized inside the nuclei and nucleolus as well as in the cytoplasm of midgut cells (Figure 4A-F) and both inside and outside the nuclei in bacteriocyte cells (Figure 5A-H). Sodalis was not detected in the ovaries/testes or salivary glands (not shown). The immunolocalization of Sodalis using mouse anti-Sodalis glossinidius GroEL monoclonal antibody also confirmed its endonuclear location inside midgut cells (Figure 6A,B). Attempts were made to examine if CLso co-localized with Sodalis, which may imply Sodalis-CLso interactions within B. trigonica. As evidenced by Figure S1A-D, the bacteria did not co-localize; CLso localized in a predicted stripe-like pattern within the midgut cells (Figure S1C,D), as observed previously in our work with Candidatus Liberibacter asiaticus (CLas) in the Asian citrus psyllid (ACP) Diaphorina citri $(16,28)$.
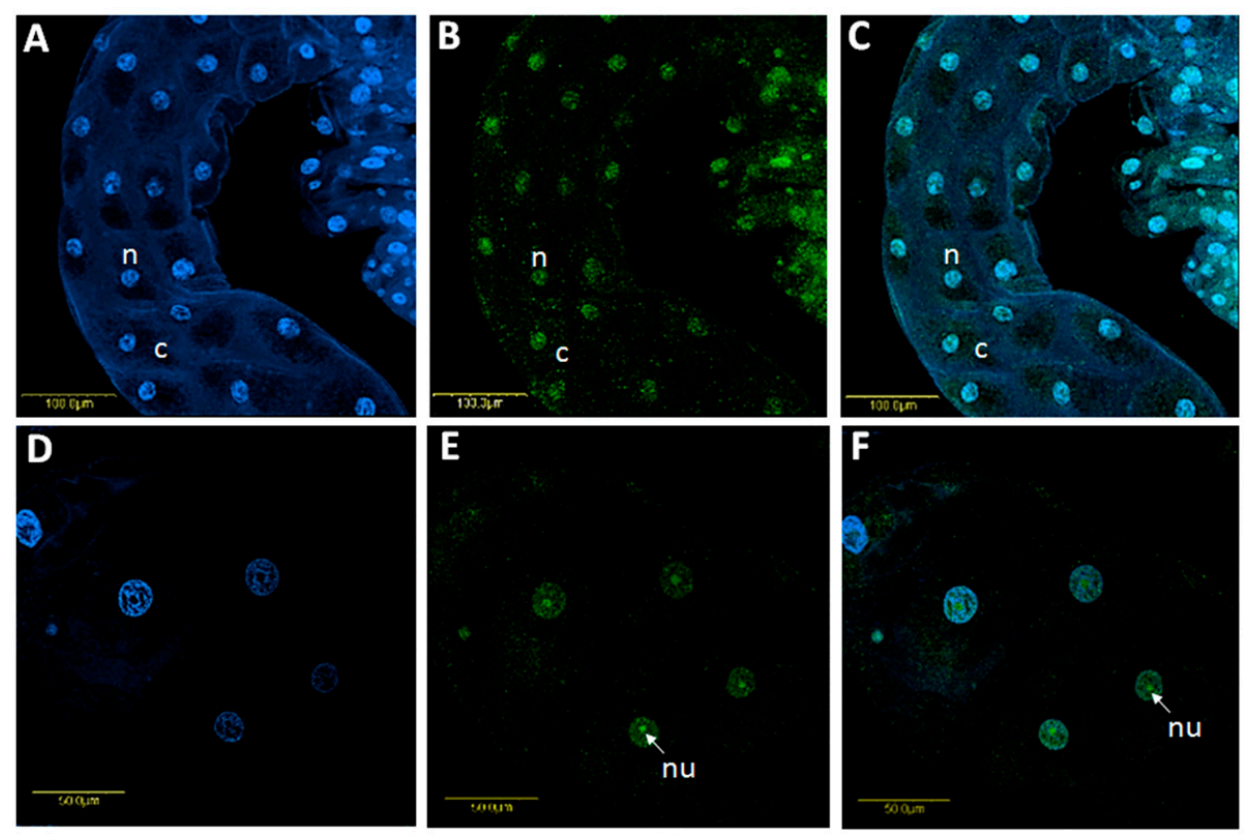

Figure 4. Localization of Sodalis in B. trigonica midgut using fluorescent in situ hybridization (FISH) with a Sodalis-specific probe. A and D: DAPI staining of BT midgut nuclei (blue). B and E: Localization of Sodalis (green) inside B. trigonica midguts by FISH, mostly showing specific localization inside the nucleus and the nucleolus and some localization in the cytoplasm around the nucleus. $\mathbf{C}$ and F: Overlay of Sodalis and DAPI staining inside midgut nuclei under a bright field. Arrows indicate, n: nucleus; nu: nucleolus; c: cytoplasm. 

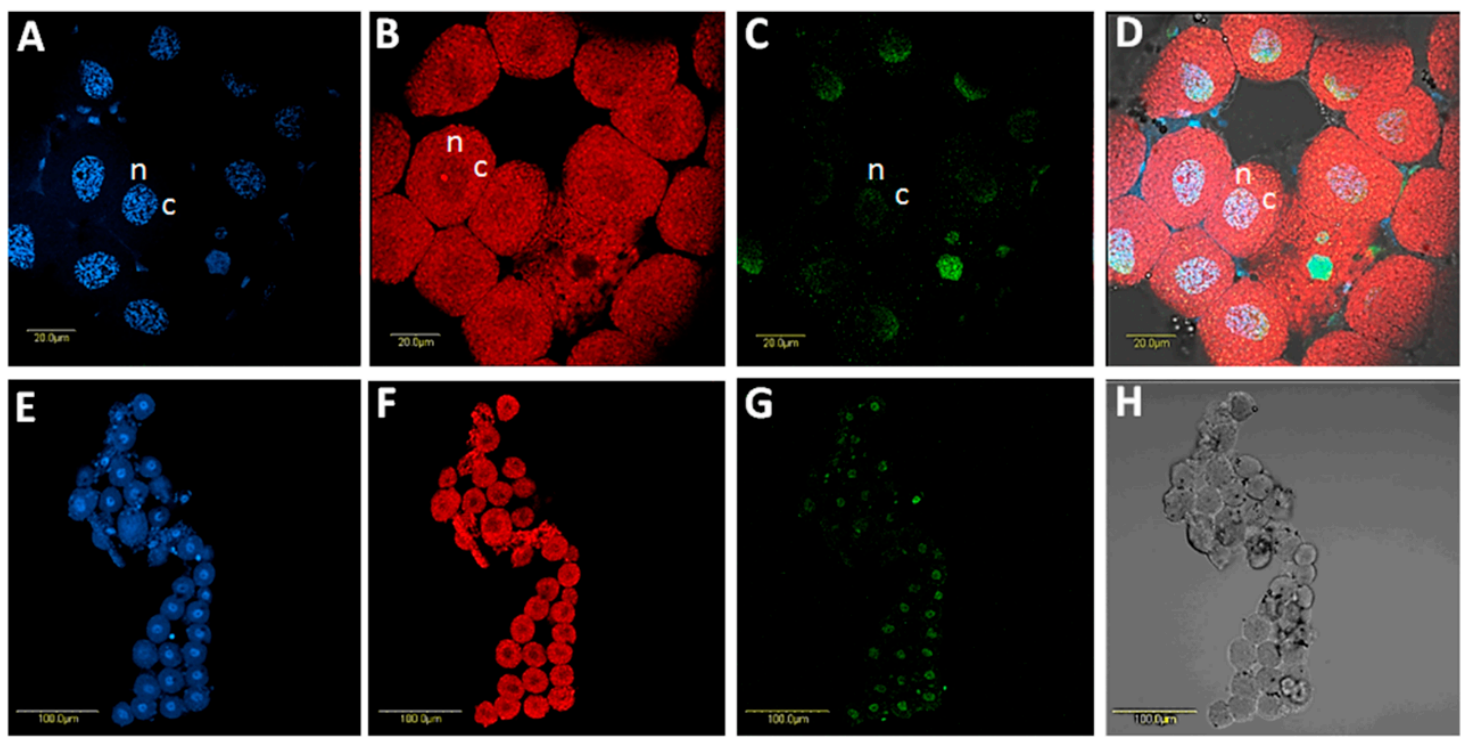

Figure 5. Localization of Sodalis in B. trigonica bacteriocytes using FISH with Sodalis-specific probe. A, DAPI-stained bacteriocyte nuclei. B, Same photo as in A showing the localization of Carsonella, the primary symbiont of psyllids (red) in the bacteriocytes. C, Sodalis localization (green) inside bacteriocyte nuclei. D, Overlay of A, B and C under a bright field. E-G show a cluster of bacteriocytes with DAPI, Carsonella and Sodalis staining, respectively. H shows the same cluster of bacteriocytes under a bright field. n: nucleus; c: cell cytoplasm.
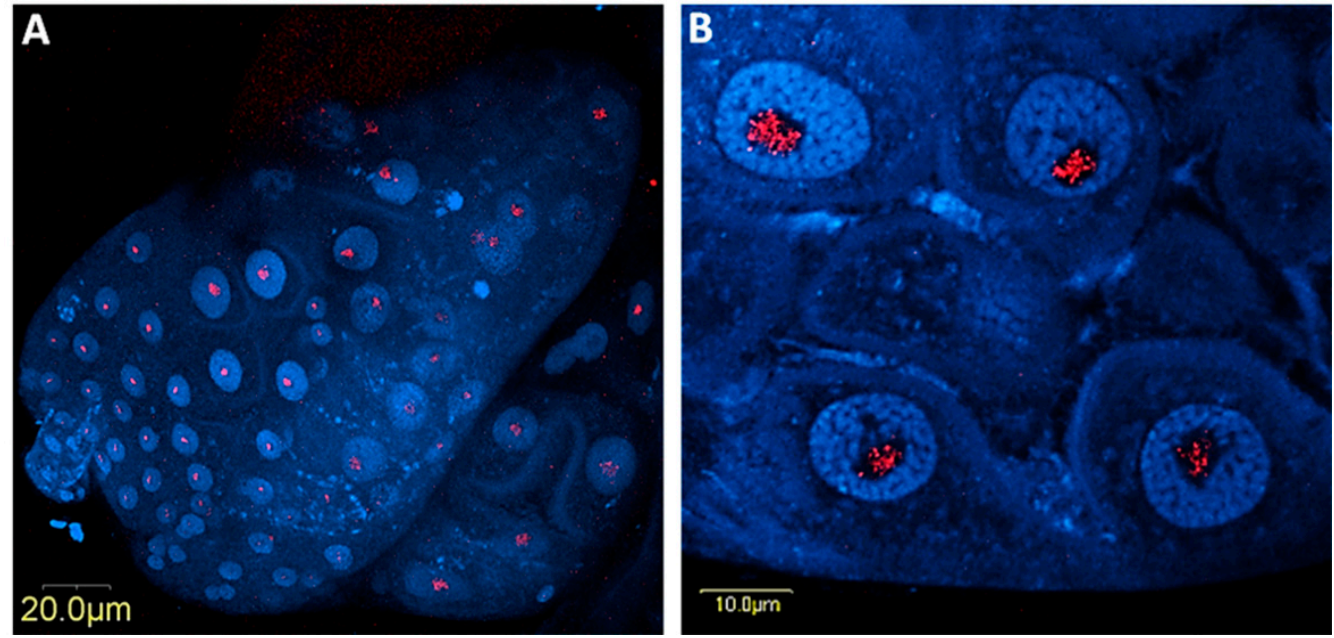

Figure 6. Immunolocalization of Sodalis (red) in B. trigonica midguts using anti-Sodalis monoclonal antibodies and cy3 labelled anti-mouse secondary antibody. The midgut nuclei were stained with DAPI (blue). A, the posterior midgut and $\mathbf{B}$, in higher magnification showing four nuclei with Sodalis localized inside (red).

Spiroplasma localization was restricted to the midgut cell cytoplasm (Figure 7A,B,E,F) using FISH, and DNA was not detected in bacteriocytes (Figure 7G,H). Spiroplasma appeared to aggregate in patches around the nucleus using a specific fluorescent probe and under UV light to visualize the DAPI staining of the nuclei. The Spiroplasma patches seemed to segregate with cell division and were included in the dividing cells (Figure 7A,B,E,F). The co-localization of Spiroplasma with CLso, as seen in Figure 7A-D,F, was not observed. CLso showed the same stripe-like patterns as before but did not co-localize with Spiroplasma in the cell cytoplasm. 

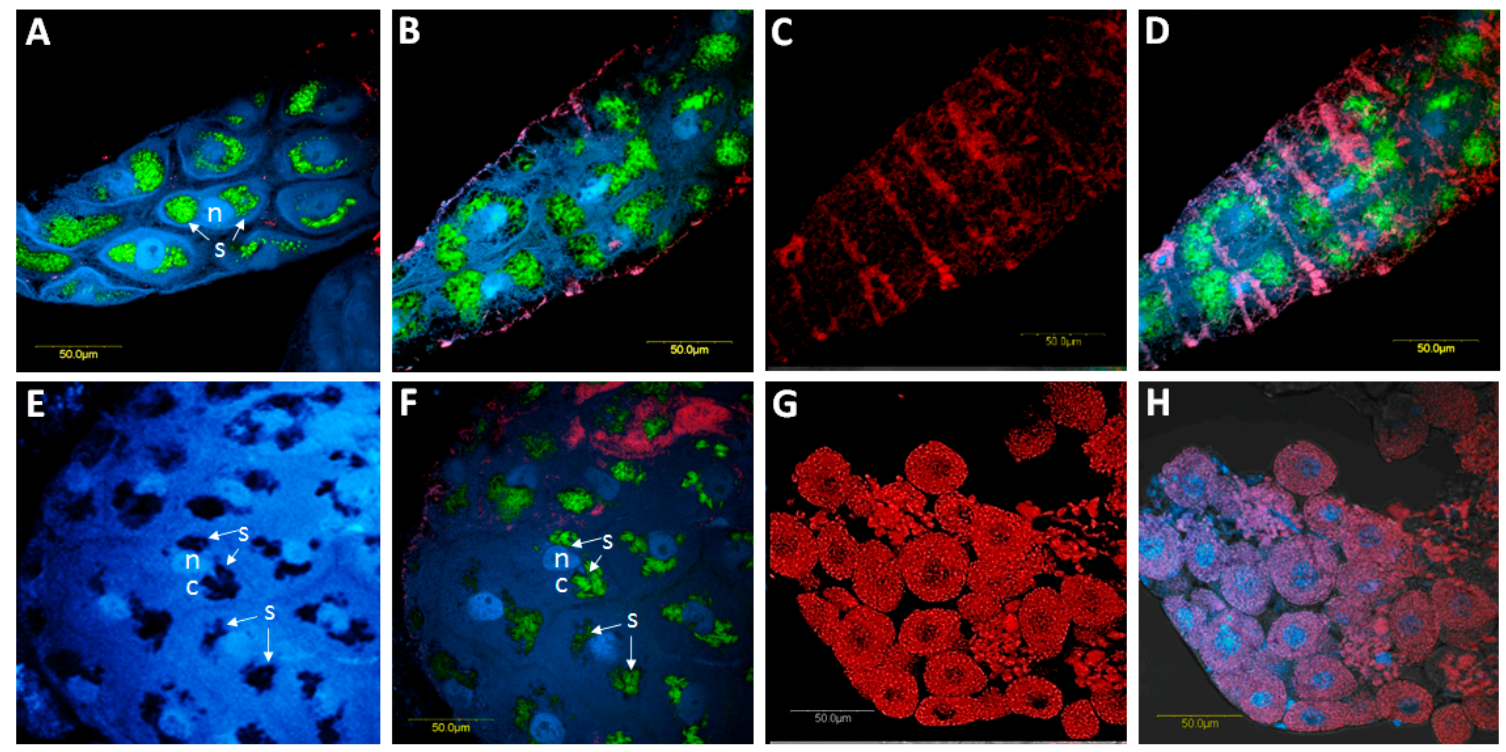

Figure 7. Co-localization of Spiroplasma and CLso in the B. trigonica midgut using FISH. A, B, focal plans showing the localization of Spiroplasma (green) in BT midguts by FISH and DAPI-stained nuclei (blue). In these focal planes, some localization of CLso appears (red). C, the same portion of the midgut in B showing the localization of CLso (red). D, overlay of Spiroplasma, midgut nuclei and CLso under bright field. E-F, other views of Spiroplasma localization (green) and DAPI-stained nuclei (blue). E, Spiroplasma patches around the nuclei without any staining. F, Spiroplasma patches with staining. G, FISH using Carsonella (red) and Spiroplasma probes in bacteriocytes. Spiroplasma was not detected inside bacteriocyte cells. $\mathbf{H}$ is the overlay of Carsonella, Spiroplasma and DAPI-stained nuclei in bacteriocytes. n: nucleus; c: cellular cytoplasm. Arrows indicate, s: Spiroplasma.

\subsection{Sequence of the Sodalis-Like Endosymbiont of Bactericera trigonica}

Using the Illumina assembly, we produced a preliminary draft sequence for Sodalis, which was used to characterize the basic genomic properties and metabolic functions of this bacterium. The sequence length of the draft assembly of Sodalis was $1.58 \mathrm{Mb}$ with $55.7 \%$ GC content. The draft genome consisted of 746 scaffolds with an N50 of $2843 \mathrm{bp}$. Phylogenetic analysis using orthologous genes from all available Sodalis genome sequences re-clustered the Sodalis endosymbiont from $B$. trigonica with that of Sodalis glossinidius, the secondary symbiont found in tsetse flies (Figure 8). However, the B. trigonica-associated Sodalis genome size was reduced by more than 2.7 times compared to Sodalis glossinidius $(4.29 \mathrm{Mb})$, and the former lacks most orthologous genes across functional categories such as biosynthetic pathways for most amino acids, vitamin biosynthesis and carbohydrate utilization.

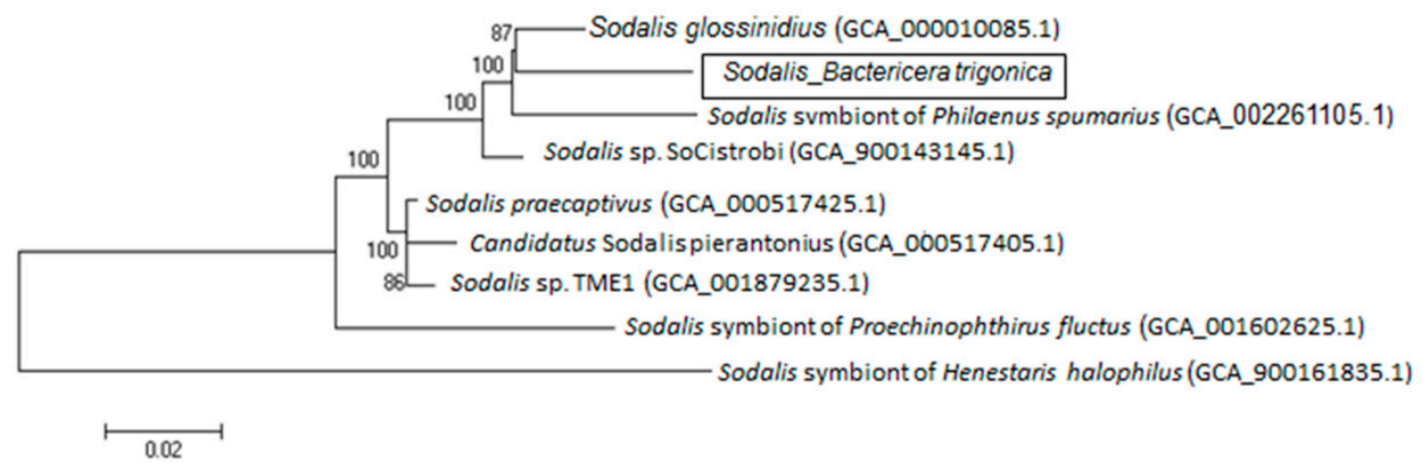

Figure 8. Phylogeny of 3404 orthologous genes (concatenated sequences of 347,000 bp each) of the Sodalis sp genomes available from the GenBank including the Sodalis identified from B. trigonica. 


\section{Discussion}

Symbiont diversity and localization inside their insect hosts have immense ecological importance, with potential downstream applications on vector control. In this study, we characterized two endosymbiotic bacteria that co-habit field populations of the carrot psyllid Bactericera trigonica. Sodalis $s p$ are Gram-negative bacterial endosymbionts commonly associated with many insects such as tsetse flies [40], weevils [41], stink bugs [42], beetles [43], aphids [44], louse flies [45] and even some psyllid species [14,17]. Although Sodalis has been previously detected in other psyllid species [17], such as the potato psyllid (Bactericera cockerelli), eucalyptus psyllid (Blastopsylla occidentalis) and the alder psyllid (Psylla floccosa), the infection prevalence of Sodalis in these insect species vary considerably. Sodalis was detected in $100 \%$ of the B. trigonica samples tested in Israel. We expected that Sodalis infecting B. trigonica would be closely related to Sodalis-like species that infect other psyllid hosts. However, to our surprise, the phylogenetic analysis of the $16 \mathrm{~s}$ rDNA nucleotide sequence revealed the highest similarity to Sodalis sp., infecting stink bugs and weevils but not with those infecting other closely related hemipterans such as psyllids, aphids and mealybugs. The $16 \mathrm{~S}$ rDNA sequence of Sodalis infecting B. trigonica was greater than $98 \%$, which was similar to that of Sodalis praecaptivus (a pathogenic isolate from a human wound) and Sodalis glossinidius, a secondary symbiont in tsetse flies. It is possible that the Sodalis associated with B. trigonica has been recently acquired from Sodalis-like symbionts that colonize other insect species via horizontal transfer. Since S. glossinidius [40,46] and S. praecaptivus [47] can be cultured in vitro, it has been suggested that these Sodalis strains may contribute to an environmental pool for symbiont acquisition [41].

Spiroplasma was found to infect $82.5 \%$ of B. trigonica and is closely related to Spiroplasma ixodetis. How Spiroplasma was acquired by B. trigonica remains unknown, but this is maintained in psyllids through vertical transfer from parents to offspring. Spiroplasma species closely related to S. ixodetis are known to induce male killing in butterfly and ladybird beetle larvae [48]. It remains unknown whether the strain of Spiroplasma that infects B. trigonica can produce a similar phenotype. However, the exclusive localization of Spiroplasma in the adult midgut tissue of $B$. trigonica limits its potential for reproductive manipulation. Wolbachia and Arsenophonus, the other two predominant secondary symbiont species known to infect other psyllid species, were not detected in B. trigonica collected from our field sites. Moreover, in this study, we also estimated the prevalence of recently described densovirus (BtDNV) infecting B. trigonica [27]. BtDNV was detected in $100 \%$ of the psyllid samples tested. Interestingly, adult psyllids infected with CLso had significantly reduced titers of BtDNV compared to CLso-uninfected psyllids. Densovirus titers inside insects have been previously shown to be influenced by the presence of viruses transmitted by the insect [49] or other symbionts [50]. The reasons for reduced titers of BtDNV inside CLso-infected psyllids remain unknown but could be due to competitive interactions between BtDNV and CLso inside the psyllid.

Sodalis inside B. trigonica occupy an unusual intranuclear niche within midgut cells, as confirmed by FISH and immunostaining using a Sodalis-specific monoclonal antibody. However, we unsuccessfully attempted to visualize the intranuclear habitat of Sodalis inside the midgut cells by TEM. The possible reasons for this failure possibly included the low titers of Sodalis, which make it difficult to visualize Sodalis inside the densely stained nuclei of the midgut cells. Intranuclear symbiosis is a rare phenomenon, and only Rickettsia and Orientia-like organisms have been reported to inhabit to insect cell nuclear organelles [51]. Hemipteran insects harbor Rickettsia-like bacteria inside Malpighian tubule nuclei, midgut cells, salivary glands and ovaries [52-54]. This is the first time a species of Sodalis has been reported to colonize the nuclei of insect cells, and the evolutionary reason for such compartmentalization is intriguing. Intranuclear location might confer advantages to symbionts such as protection from cytoplasmic defense mechanisms. The nucleus also provides a rich pool of proteins and nucleic acid resources for symbiont growth [51]. Eukaryotic nuclei control gene regulation and cell division. Thus, intracellular pathogenic bacteria can selectively target the host cell nucleus to subvert host defenses by releasing effector molecules, which enter the host cell nucleus and hijack nuclear processes [55]. Thus, the presence of Sodalis inside the midgut cell nuclei allows room for the direct manipulation of the 
host cell by targeting important nuclear processes including CLso establishment. FISH analyses of infected nuclei did not exhibit any co-localization of Sodalis with Spiroplasma or CLso in the psyllid midguts, which suggests that any influence these two microorganisms have on the host-pathogen interactions will likely be indirect. Sodalis sp. can facilitate pathogen establishment in insect vectors; for example, it has been proposed that Sodalis glossinidius synthesizes exochitinases, which degrade chitin and produce lectin-inhibitory sugars. These sugars may help trypanosomatid parasites establish in the tsetse fly (vector) midgut by inhibiting the production of trypanocidal midgut lectins [40,56]. Whether Sodalis inside $B$. trigonica also conditions the psyllid for CLso acquisition in similar ways remains unknown; however, a complete pathway for $\beta$-galactosidase synthesis remains intact. Unfortunately, the functional activity of Sodalis on CLso establishment could not be tested in this study due to the limitation of acquiring Sodalis-free psyllids as $100 \%$ of the field isolates contained this symbiont.

Paratransgenesis is a vector control technology that involves the manipulation of an insect phenotype by expression from a transformed symbiont [57]. Both Sodalis and BtDNV are good candidates for paratransgenesis due to their multiple-insect host range, efficient vertical transmission to offspring and amenability to genetic transformation. Recombinant Sodalis glossinidius was engineered to express anti-trypanosomal nano-antibodies to limit trypanosome development in tsetse midguts [58,59]. The in vivo proximity of Sodalis to CLso within the psyllid midgut and its localization in the nucleus are two attributes that increase its potential for delivering foreign DNA into the host cell or constitutively expressing dsRNA to disrupt CLso establishment and transmission. The successful, robust in vitro culture of Sodalis from B. trigonica and the re-introduction of a recombinant Sodalis into the psyllid are obstacles which are yet to be overcome before we can constitutively express dsRNA to disrupt CLso establishment in the insect gut. However, the ability to culture Sodalis ex insecta has not yet been resolved. In-depth genome analysis will help shed light on the possibility of its independent existence outside host cells and which key medium additives are crucial for growth. The preliminary draft genome of the Sodalis associated with B. trigonica, described in this study, reveals a heavily reduced genome size of $1.57 \mathrm{Mb}$ compared to eight other Sodalis genomes isolated from different insect species (with a genome size range from 1.3 to $5.1 \mathrm{Mb}$ ). This truncated genome might be an indication of a higher dependency on its insect host, thus limiting its cell-free existence, as Sodalis species that have been cultured in vitro tend to have larger genomes (> $4 \mathrm{MB})$.

Accession numbers of sequences generated in this study:

- $\quad$ Sodalis draft genome - GCA_003668825.1

- $\quad$ Sodalis 16S rDNA - MH973240, MH973241

- $\quad$ Sodalis partial GroEL - MH987777

- Spiroplasma 16S rDNA - MH973257

- $\quad$ CLso 16S rDNA - MH986752

Supplementary Materials: The following are available online at http://www.mdpi.com/2076-2607/8/5/692/s1, Figure S1: Localization of Sodalis and CLso using FISH with specific probes in B. trigonica midgut tissue 10 days after CLso acquisition. A, DAPI staining (blue) of B. trigonica midgut nuclei. B, Localization of Sodalis (green) in the same midgut seen in (A) but showing very faint endonuclear localization. C, Localization of CLso (red) in the same midgut seen in A and B. D, Overlay of Sodalis, CLso and midgut nuclei under bright field.

Author Contributions: S.G. designed and performed experiments and prepared the original draft of the manuscript. N.S. performed the bioinformatic analysis. G.L. and S.K. helped in performing the experiments and in maintaining and providing insects. L.R.H. designed experiments, provided antibodies, drafted the manuscript and discussed the results. M.G. raised the funding, designed experiments, and wrote and submitted the final version of the manuscript. All authors have read and agreed to the published version of the manuscript.

Funding: This research was supported by grant number 1163/18 from Israel Science Foundation (ISF) to M.G.

Conflicts of Interest: The authors declare no conflict of interest. The funders had no role in the design of the study; in the collection, analyses, or interpretation of data; in the writing of the manuscript, or in the decision to publish the results. 


\section{References}

1. Moran, N.A.; Baumann, P. Bacterial endosymbionts in animals. Curr. Opin. Microbiol. 2000, 3, $270-275$. [CrossRef]

2. Baumann, P. Biology of bacteriocyte-associated endosymbionts of plant sap-sucking insects. Annu. Rev. Microbiol. 2005, 59, 155-189. [CrossRef] [PubMed]

3. Wilson, A.C.C.; Duncan, R.P. Signatures of host/symbiont genome coevolution in insect nutritional endosymbioses. Proc. Natl. Acad. Sci. USA 2015, 112, 10255-10261. [CrossRef] [PubMed]

4. Baumann, P.; Moran, N.A.; Baumann, L.; Dworkin, M. Bacteriocyte-associated endosymbionts of insects. Prokaryotes 2006, 1, 403-438.

5. Montllor, C.B.; Maxmen, A.; Purcell, A.H. Facultative bacterial endosymbionts benefit pea aphids Acyrthosiphon pisum under heat stress. Ecol. Entomol. 2002, 27, 189-195. [CrossRef]

6. Brumin, M.; Kontsedalov, S.; Ghanim, M. Rickettsia influences thermotolerance in the whitefly Bemisia tabaci B biotype. Insect Sci. 2011, 18, 57-66. [CrossRef]

7. Łukasik, P.; Guo, H.; Asch, M.; Ferrari, J.; Godfray, H.C.J. Protection against a fungal pathogen conferred by the aphid facultative endosymbionts Rickettsia and Spiroplasma is expressed in multiple host genotypes and species and is not influenced by co-infection with another symbiont. J. Evol. Biol. 2013, 26, 2654-2661. [CrossRef]

8. Hendry, T.A.; Hunter, M.S.; Baltrus, D.A. The Facultative Symbiont Rickettsia Protects an Invasive Whitefly against Entomopathogenic Pseudomonas syringae Strains. Appl. Environ. Microbiol. 2014, 80, 7161-7168. [CrossRef]

9. Oliver, K.M.; Russell, J.A.; Moran, N.A.; Hunter, M.S. Facultative bacterial symbionts in aphids confer resistance to parasitic wasps. Proc. Natl. Acad. Sci. 2003, 100, 1803-1807. [CrossRef]

10. Kontsedalov, S.; Zchori-Fein, E.; Chiel, E.; Gottlieb, Y.; Inbar, M.; Ghanim, M. The presence of Rickettsia is associated with increased susceptibility of Bemisia tabaci (Homoptera: Aleyrodidae) to insecticides. Pest Manag. Sci. 2008, 64, 789-792. [CrossRef]

11. Himler, A.G.; Adachi-Hagimori, T.; Bergen, J.E.; Kozuch, A.; Kelly, S.E.; Tabashnik, B.E.; Chiel, E.; Duckworth, V.E.; Dennehy, T.J.; Zchori-Fein, E.; et al. Rapid spread of a bacterial symbiont in an invasive whitefly is driven by fitness benefits and female bias. Science 2011, 332, 254-256. [CrossRef] [PubMed]

12. Gottlieb, Y.; Zchori-Fein, E.; Mozes-Daube, N.; Kontsedalov, S.; Skaljac, M.; Brumin, M.; Sobol, I.; Czosnek, H.; Vavre, F.; Fleury, F. The transmission efficiency of tomato yellow leaf curl virus by the whitefly Bemisia tabaci is correlated with the presence of a specific symbiotic bacterium species. J. Virol. 2010, 84, 9310-9317. [CrossRef] [PubMed]

13. Kliot, A.; Cilia, M.; Czosnek, H.; Ghanim, M. Implication of the bacterial endosymbiont Rickettsia spp. in interactions of the whitefly Bemisia tabaci with Tomato yellow leaf curl virus. J. Virol. 2014, 88, 5652-5660. [CrossRef] [PubMed]

14. Thao, M.L.; Clark, M.A.; Baumann, L.; Brennan, E.B.; Moran, N.A.; Baumann, P. Secondary endosymbionts of psyllids have been acquired multiple times. Curr. Microbiol. 2000, 41, 300-304. [CrossRef] [PubMed]

15. Nakabachi, A.; Ueoka, R.; Oshima, K.; Teta, R.; Mangoni, A.; Gurgui, M.; Oldham, N.J.; van Echten-Deckert, G.; Okamura, K.; Yamamoto, K. Defensive bacteriome symbiont with a drastically reduced genome. Curr. Biol. 2013, 23, 478-1484. [CrossRef] [PubMed]

16. Subandiyah, S.; Nikoh, N.; Tsuyumu, S.; Somowiyarjo, S.; Fukatsu, T. Complex endosymbiotic microbiota of the citrus psyllid Diaphorina citri (Homoptera: Psylloidea). Zoolog. Sci. 2000, 17, 983-989. [CrossRef]

17. Spaulding, A.W.; von Dohlen, C.D. Psyllid endosymbionts exhibit patterns of co-speciation with hosts and destabilizing substitutions in ribosomal RNA. Insect Mol. Biol. 2001, 10, 57-67. [CrossRef]

18. Arp, A.; Munyaneza, J.E.; Crosslin, J.M.; Trumble, J.; Bextine, B. A global comparison of Bactericera cockerelli (Hemiptera: Triozidae) microbial communities. Environ. Entomol. 2014, 43, 344-352. [CrossRef]

19. Antolínez, C.A.; Fereres, A.; Moreno, A. Sex-specific probing behaviour of the carrot psyllid Bactericera trigonica and its implication in the transmission of 'Candidatus Liberibacter solanacearum'. Eur. J. Plant Pathol. 2017, 147, 627-637.

20. Antolinez, C.A.; Fereres, A.; Moreno, A. Risk assessment of 'Candidatus Liberibacter solanacearum'transmission by the psyllids Bactericera trigonica and B. tremblayi from Apiaceae crops to potato. Sci. Rep. 2017, 7. [CrossRef] 
21. Ghanim, M.; Achor, D.; Ghosh, S.; Kontsedalov, S.; Lebedev, G.; Levy, A. 'Candidatus Liberibacter asiaticus' Accumulates inside Endoplasmic Reticulum Associated Vacuoles in the Gut Cells of Diaphorina citri. Sci. Rep. 2017, 7, 16945. [CrossRef] [PubMed]

22. Ghosh, S.; Jassar, O.; Kontsedalov, S.; Lebedev, G.; Wang, C.; Donielle, T.; Levi, A.; Ghanim, M. A transcriptomics approach reveals putative interaction of Candidatus Liberibacter solanacearum with the Endoplasmic Reticulum of its psyllid vector. Insects 2019, 10, 279. [CrossRef] [PubMed]

23. Mawassi, M.; Dror, O.; Bar-Joseph, M.; Piasetzky, A.; Sjölund, J.; Levitzky, N.; Shoshana, N.; Meslenin, L.; Haviv, S.; Porat, C. 'Candidatus Liberibacter solanacearum' is Tightly Associated with Carrot Yellows Symptoms in Israel and Transmitted by the Prevalent Psyllid Vector Bactericera trigonica. Phytopathology 2018, 108, 1056-1066. [CrossRef] [PubMed]

24. Van den Heuvel, J.F.; Bruyere, A.; Hogenhout, S.A.; Ziegler-Graff, V.; Brault, V.; Verbeek, M.; Van Der Wilk, F.; Richards, K. The N-terminal region of the luteovirus readthrough domain determines virus binding to Buchnera GroEL and is essential for virus persistence in the aphid. J. Virol. 1997, 71, 7258-7265. [CrossRef]

25. Walker, T.; Johnson, P.H.; Moreira, L.A.; Iturbe-Ormaetxe, I.; Frentiu, F.D.; McMeniman, C.J.; Leong, Y.S.; Dong, Y.; Axford, J.; Kriesner, P. The wMel Wolbachia strain blocks dengue and invades caged Aedes aegypti populations. Nature 2011, 476, 450-453. [CrossRef]

26. McMeniman, C.J.; O'Neill, S.L. A virulent Wolbachia infection decreases the viability of the dengue vector Aedes aegypti during periods of embryonic quiescence. PLoS Negl. Trop Dis. 2010, 4, e748. [CrossRef]

27. Ghosh, S.; Sela, N.; Ghanim, M. Complete Genome Sequence of a Putative Densovirus Infecting the Carrot Psyllid Bactericera trigonica. Microbiol. Resour. Announc. 2019. [CrossRef]

28. Shahjahan, R.; Hughes, K.; Leopold, R.; Devault, J. Lower incubation temperatures increase yield of insect genomic DNA isolated by the CTAB method. Biotechniques 1995, 19, 332-334.

29. Weisburg, W.G.; Barns, S.M.; Pelletier, D.A.; Lane, D.J. 16 S ribosomal DNA amplification for phylogenetic study. J. Bacteriol. 1991, 173, 697-703. [CrossRef]

30. Ishii, K.; Takii, S. Comparison of microbial communities in four different composting processes as evaluated by denaturing gradient gel electrophoresis analysis. J. Appl. Microbiol. 2003, 95, 109-119. [CrossRef]

31. Greuter, D.; Loy, A.; Horn, M.; Rattei, T. probeBase-an online resource for rRNA-targeted oligonucleotide probes and primers: new features 2016. Nucleic. Acids Res. 2016, 44, D586-D589. [CrossRef] [PubMed]

32. Ghanim, M.; Fattah-Hosseini, S.; Levy, A.; Cilia, M. Morphological abnormalities and cell death in the Asian citrus psyllid (Diaphorina citri) midgut associated with Candidatus Liberibacter asiaticus. Sci. Rep. 2016, 6, 33418. [CrossRef] [PubMed]

33. Rose, C.; Belmonte, R.; Armstrong, S.D.; Molyneux, G.; Haines, L.R.; Lehane, M.J.; Wastling, J.; Acosta-Serrano, A. An investigation into the protein composition of the teneral Glossina morsitans morsitans peritrophic matrix. PLoS Negl. Trop Dis. 2014, 8, e2691. [CrossRef] [PubMed]

34. Bolger, A.M.; Lohse, M.; Usadel, B. Trimmomatic: a flexible trimmer for Illumina sequence data. Bioinformatics 2014, 30, 2114-2120. [CrossRef]

35. Tritt, A.; Eisen, J.A.; Facciotti, M.T.; Darling, A.E. An integrated pipeline for de novo assembly of microbial genomes. PLoS ONE 2012, 7, e42304. [CrossRef]

36. Lu, C.L.; Chen, K.-T.; Huang, S.-Y.; Chiu, H.-T. CAR: contig assembly of prokaryotic draft genomes using rearrangements. BMC Bioinform. 2014, 15, 381. [CrossRef]

37. Aziz, R.K.; Bartels, D.; Best, A.A.; DeJongh, M.; Disz, T.; Edwards, R.A.; Formsma, K.; Gerdes, S.; Glass, E.M.; Kubal, M. The RAST Server: rapid annotations using subsystems technology. BMC Genom. 2008, 9, 75. [CrossRef]

38. Emms, D.M.; Kelly, S. OrthoFinder: solving fundamental biases in whole genome comparisons dramatically improves orthogroup inference accuracy. Genome Biol. 2015, 16, 157. [CrossRef]

39. Guindon, S.; Dufayard, J.-F.; Lefort, V.; Anisimova, M.; Hordijk, W.; Gascuel, O. New algorithms and methods to estimate maximum-likelihood phylogenies: assessing the performance of PhyML 3.0. Syst. Biol. 2010, 59, 307-321. [CrossRef]

40. Dale, C.; Maudlin, I. Sodalis gen. nov. and Sodalis glossinidius sp. nov., a microaerophilic secondary endosymbiont of the tsetse fly Glossina morsitans morsitans. Int. J. Syst. Evol. Microbiol. 1999, 49, 267-275. [CrossRef] 
41. Toju, H.; Tanabe, A.S.; Notsu, Y.; Sota, T.; Fukatsu, T. Diversification of endosymbiosis: replacements, co-speciation and promiscuity of bacteriocyte symbionts in weevils. ISME J. 2013, 7, 1378. [CrossRef] [PubMed]

42. Hosokawa, T.; Kaiwa, N.; Matsuura, Y.; Kikuchi, Y.; Fukatsu, T. Infection prevalence of Sodalis symbionts among stinkbugs. Zool. Lett. 2015, 1, 5. [CrossRef] [PubMed]

43. Grünwald, S.; Pilhofer, M.; Höll, W. Microbial associations in gut systems of wood-and bark-inhabiting longhorned beetles [Coleoptera: Cerambycidae]. Syst. Appl. Microbiol. 2010, 33, 25-34. [CrossRef] [PubMed]

44. Burke, G.R.; Normark, B.B.; Favret, C.; Moran, N.A. Evolution and diversity of facultative symbionts from the aphid subfamily Lachninae. Appl. Environ Microbiol. 2009, 75, 5328-5335. [CrossRef]

45. Nováková, E.; Husník, F.; Šochová, E.; Hypša, V. Arsenophonus and Sodalis symbionts in louse flies: an analogy to the Wigglesworthia and Sodalis system in tsetse flies. Appl. Environ. Microbiol. 2015, 81, 6189-6199.

46. Matthew, C.Z.; Darby, A.C.; Young, S.A.; Hume, L.H.; Welburn, S.C. The rapid isolation and growth dynamics of the tsetse symbiont Sodalis glossinidius. FEMS Microbiol. Lett. 2005, 248, 69-74. [CrossRef]

47. Clayton, A.L.; Oakeson, K.F.; Gutin, M.; Pontes, A.; Dunn, D.M.; von Niederhausern, A.C.; Weiss, R.B.; Fisher, M.; Dale, C. A novel human-infection-derived bacterium provides insights into the evolutionary origins of mutualistic insect-bacterial symbioses. PLoS Genet. 2012, 8, e1002990. [CrossRef]

48. Jiggins, F.M.; Hurst, G.D.D.; Jiggins, C.D.; vd Schulenburg, J.H.G.; Majerus, M.E.N. The butterfly Danaus chrysippus is infected by a male-killing Spiroplasma bacterium. Parasitology 2000, 120, 439-446. [CrossRef]

49. Pinheiro, P.V.; Wilson, J.R.; Xu, Y.; Zheng, Y.; Rebelo, A.R.; Fattah-Hosseini, S.; Kruse, A.; Dos Silva, R.S.; Xu, Y.; Kramer, M. Plant Viruses Transmitted in Two Different Modes Produce Differing Effects on Small RNA-Mediated Processes in Their Aphid Vector. Phytobiomes J. 2019, 3, 71-81. [CrossRef]

50. Parry, R.; Bishop, C.; De Hayr, L.; Asgari, S. Density-dependent enhanced replication of a densovirus in Wolbachia-infected Aedes cells is associated with production of piRNAs and higher virus-derived siRNAs. Virology 2019, 528, 89-100. [CrossRef]

51. Schulz, F.; Horn, M. Intranuclear bacteria: inside the cellular control center of eukaryotes. Trends Cell. Biol. 2015, 25, 339-346. [CrossRef] [PubMed]

52. Perotti, M.A.; Clarke, H.K.; Turner, B.D.; Braig, H.R. Rickettsia as obligate and mycetomic bacteria. FASEB J. 2006, 20, 2372-2374. [CrossRef] [PubMed]

53. Kuechler, S.M.; Gibbs, G.; Burckhardt, D.; Dettner, K.; Hartung, V. Diversity of bacterial endosymbionts and bacteria-host co-evolution in Gondwanan relict moss bugs (Hemiptera: Coleorrhyncha: Peloridiidae). Environ. Microbiol. 2013, 15, 2031-2042. [CrossRef] [PubMed]

54. Watanabe, K.; Yukuhiro, F.; Matsuura, Y.; Fukatsu, T.; Noda, H. Intrasperm vertical symbiont transmission. Proc. Natl. Acad. Sci. USA 2014, 111, 7433-7437. [CrossRef] [PubMed]

55. Bierne, H.; Cossart, P. When bacteria target the nucleus: The emerging family of nucleomodulins. Cell Microbiol. 2012, 14, 622-633. [CrossRef] [PubMed]

56. Welburn, S.C.; Arnold, K.; Maudlin, I.; Gooday, G.W. Rickettsia-like organisms and chitinase production in relation to transmission of trypanosomes by tsetse flies. Parasitology 1993, 107, 141-145. [CrossRef]

57. O'brochta, D.A.; Handler, A.M. Perspectives on the state of insect transgenics. In Transgenesis and the Management of Vector-Borne Diseas; Springer: New York, NY, USA, 2008; pp. 1-18.

58. De Vooght, L.; Caljon, G.; Stijlemans, B.; De Baetselier, P.; Coosemans, M.; Van Den Abbeele, J. Expression and extracellular release of a functional anti-trypanosome Nanobody ${ }^{\circledR}$ in Sodalis glossinidius, a bacterial symbiont of the tsetse fly. Microb. Cell Fact. 2012, 11, 23. [CrossRef]

59. De Vooght, L.; Caljon, G.; De Ridder, K.; Van Den Abbeele, J. Delivery of a functional anti-trypanosome nanobody in different tsetse fly tissues via a bacterial symbiont, Sodalis glossinidius. Microb. Cell Fact. 2014, 13, 156. [CrossRef]

(C) 2020 by the authors. Licensee MDPI, Basel, Switzerland. This article is an open access article distributed under the terms and conditions of the Creative Commons Attribution (CC BY) license (http://creativecommons.org/licenses/by/4.0/). 